\title{
Towards an Embedded and Real-Time Joint Human-Machine Monitoring Framework: Dataset Optimization Techniques for Anomaly Detection
}

\author{
Rafaella Elia, George Plastiras and Theocharis Theocharides \\ Department of Electrical and Computer Engineering and KIOS Research and Innovation Center of Excellence \\ University of Cyprus, Nicosia, Cyprus \\ \{relia001, gplast01, ttheocharides\}@ucy.ac.cy
}

\begin{abstract}
Unmanned Remotely Operated Vehicles (ROVs) are widely used across many civil application domains including real-time monitoring, security and surveillance, and search and rescue missions. Most of these applications require the human operator to control the ROV under stressful conditions and harsh environments. As such, the remote-control operator is prone to sometimes issuing anomalous commands, because of either unwanted hand or finger motion or even irrational decisions, results of fatigue, stress, etc. To enable detection of such anomalies, we propose the use of a joint human-ROV monitoring framework, by monitoring the human operator's bio-signals and the ROV's sensory data. The framework is anticipated to run on the ROV, enabling it to recognize and possibly ignore anomalies, potentially paving the way for a shared control algorithm. In this paper therefore, we present a first step towards achieving this goal, focusing on optimizing the fused dataset consisting of the aforementioned signals and investigated different techniques such as feature extraction and statistical component analysis, in an effort to reduce the dimensionality of the dataset. To this end, we present a dataset constructed by surface Electromyography (sEMG) signals from various operators, fused with the ROV's inertial sensors. Through our proposed optimizations, we are able to reduce both the data size as well as the necessary features and signal components, while maintaining the ability to detect anomalies with at least $85 \%$ accuracy depending on the dimensionality reduction technique (over raw data). We evaluated our dataset over a variety of classifier configurations and embedded platforms with noteworthy energy and performance benefits.
\end{abstract}

Index Terms-Remotely Operated Vehicles (ROVs), Anomaly Detection, Embedded and Real Time Systems

\section{INTRODUCTION}

Unmanned Remotely Operated Vehicles (ROVs) are widely used across many civil application domains including real-time monitoring, security and surveillance, and search and rescue missions. Most of these applications require the human operator to control the ROV under stressful conditions and harsh environments [1]. Operating in extreme conditions, dealing with the scarcity of human resources and having the feeling of urgency, makes the human operator nervous which implies the risk of not being able to perform the mission, or to crash the ROV. Due to the fact of an increased stress and workload during difficult and demanding missions, the operator may make some unintentional movements, which can lead to an unwanted result. Monitoring a ROV [2], [3] or the human operator [1], [4], [5], has been heavily researched and there is a variety of solutions and approaches. However, there are situations where there is a need for a controlling mechanism emdebbed on the ROV to prevent accidents that may occur due to an inexperience or stressed pilot. Characterizing the mental or physical state of the operator in critical missions is extremely important and can prevent accidents [1]. Preventing and externally controlling unwanted commands, can be easily implemented using non-invasive wearable sensors for physiological signal monitoring along with an embedded mechanism controlling or assisting the operator. However, looking at the human operator solely, may result into restrictive models that might fail to accurately capture the state of the operator. On the other hand, designing a mechanism to jointly monitoring the human and the ROV can lead to a massive amount of data that needs processing. As a result this can be translated into huge memory requirement, processing time and power consumption. If we assume that such a mechanism will be deployed on the ROV, problem is getting harder if the monitoring framework will require communication with the ROV controlling system. Transmitting a significant amount of information can be time consuming and in an emergency situation this is not desirable. We need a resource-constrained and low-energy framework to act as a shared control mechanism for monitoring the human and the ROV in search and rescue missions or any similar emergency situation. To this end, we take a step towards this direction through the contributions of our work:

- We construct a dataset from experienced and non-experienced ROV operators, consisting of surface Electromyography (sEMG) signals and the ROV's inertial sensors data during operation.

- We investigate dimensionality reduction techniques to optimize the dataset and to reduce the computation and communication overheads to achieve real-time classification of normal and abnormal movements.

- We evaluate our dataset towards the classification of normal and abnormal movements using various classifiers implemented on resource-contrained platforms.

- We perform a design-space exploration on the impact of our dataset optimizations on the accuracy, performance and energy for real-time classification on embedded platforms.

The rest of the paper is organized as follows. Section II presents an overview of existing similar approaches and the fundamental background. Section III describes the development of our dataset. Section IV describes the feature extraction and dimensionality reduction techniques and Section V describes our experimental results evaluated across various classifiers and implemented on three embedded platforms with different characteristics. Section VI concludes the paper and presents directives for future work. 


\section{THEORETICAL BACKGROUND AND RELATED WORK}

\section{A. Sensing the Human Operator}

There is an increasing amount of work in physiological monitoring of human operators, through various non-invasive techniques and sensors [1], [4], [5]. Physiological signals provide us with important information about the human mental or physical state, that they cannot be detected simply from facial expressions, voice tone, body movements, etc. There is a plethora of such sensors measuring physiological changes [1], [4], [5]. In our case, we aim in detecting anomalies caused by involuntary movements or by other extrinsic forces on the ROV, such as turbulence, etc. We thus aim to monitor the human operator via sEMG signals, fuse the sEMG signals with inertial sensor data from the ROV, and classify them correctly as normal and abnormal. Electromyogram (EMG) is a measure of muscular activity and can be represented as a function of time, defined in terms of amplitude, frequency and phase [6]. It is mainly used for the study of muscle functioning, for determining how movements are performed, as well as to monitor deformations of muscle volume.

While the detection and classification of sEMG signal is a heavily researched topic [6], [7], it has not been applied in situations where there is a need to monitor the movement of an ROV operator; further, it has not been used in conjunction with the ROV signals elsewhere. In terms of classification of sEMG signals, a recent study [7] proposes feature extraction and classification of EMG signal using Linear Discriminant Analysis. Authors make use of a vast amount and variety of features, both in time and frequency domain and they illustrate which features provide the higher accuracy for each case-movement that needs to be classified. The sEMG has been used in a variety of studies, trying to estimate workload and stress or fatigue of muscles through sEMG signal [4], [5]. We selected sEMG because is a non-invasive technique suitable for monitoring the muscle functioning and furthermore, for monitoring the finger movements of the ROV's operator.

\section{B. Remotely Operated Vehicles (ROVs)}

In terms of self-monitoring devices and machines, i.e. the ROV, there is an abundant amount of work; this is quite evident in self-awareness research, let that involving research towards self-aware systems, as well as self-aware cyberphysical systems including human-machine interfaces. Applications ranging from prosthetic control, biomedical robots, aircraft control, driver assistance systems, human-computer interfaces, and many more, feature built-in sensors that monitor the system's status as well as the human user. The majority of such works though, does not cope with resource-constraint computation, instead focuses on the transportation industry [5], and automotive and avionic systems [4], [8], [9]. In terms of our targeted framework, our previous work related to Unmanned Aerial Vehicles (UAVs) [2], describes the design of a small, intelligent, low-power, real-time and built-in controller for multi-rotor UAVs which is able to understand a dangerous scenario right before it happens, and by taking measures to keep the drone safe. Additionally, a similar work is described in [3] where authors proposed a health-management based hierarchical architecture model for multiple types of vehicle systems. The aim was to monitor and receive health management information about each mission system component, to improve the mission system's self-awareness and adapt vehicle guidance, task and mission plans. This approach was tested only between drones and a few ground vehicles, and no human was involved in the experiment.

\section{Joint Human - ROV Monitoring}

In the context of our framework, we explored studies investigating the joint human and ROV monitoring. As stated earlier, automotive and aviation industry applications get a lot of attention on this topic but they do not classify as resource constrained. In detail, authors in [9] designed a vision-based framework for driver activity recognition and prediction in overtaking situations where a camera was used for sensing the environment. Authors in [8] introduce a haptic driver vehicle steering interface which interacts with the driver through environmentally mediated torque and stiffness changes. Two types of information are provided to the driver through the steering wheel in the form of steering torques. Another approach for assistance in rescue missions with drones is described in [1]. The operator was monitored by an ECG sensor, where an ECG signal was recorded and compared to the cognitive workload estimation. The operator in this study, was driving a drone on a simulator. To the best of our knowledge, this is the first attempt for monitoring a human operator and the ROV in real environment.

\section{Contributions of this paper}

This paper aims to develop a dataset consisting of sEMG signals and ROV's inertial sensor data, suitable for detecting anomalies caused by involuntary movements, and classify them correctly. The collected data can then be used in a possibly shared-control algorithm/controller, deployed on the ROV, which will process the monitoring data in real-time and deduce when a control command issued by the operator is abnormal, possibly either ignoring it, or taking a corrective countermeasure. Moreover, we investigate dimensionality reduction techniques and compared them with the original dataset, to optimize the dataset for real-time and energy-efficient classification on resource constrained devices. We evaluate our dataset and optimization approaches using 3 well-known classifiers on embedded platforms, exploring accuracy, energy and processing time.

\section{DATASET}

\section{A. Dataset Acquisition Setup}

To construct our dataset, we used a remotely piloted drone. To form the human sEMG data part, we collected data from 6 healthy subjects, 3 males and 3 females aged 25 to 29 years old, where the 2 of them were experienced drone operators and the other 4 were non-experienced drone operators. To this end, including non-experienced operators in our study, we are able 
Table 1. Electromyography (EMG) signal features

to stimulate and capture involuntary movements under stress, similar to the movements of an experienced operator during a critical mission. Although, an experienced operator might not get stressed while operating during a normal mission, on critical missions drone operators are exposed to stressful conditions where they need to operate perfectly.Each operator was instructed to perform a pre-defined course consisting of the basic 4 simple movements (left, right, forward, backward). We collected data from the right hand only, focusing on on-air motion that is performed with a single thumb joystick. Each instructed movement was performed for $3-5$ seconds followed by a resting time of 20 seconds to avoid muscle fatigue. The flight duration varied between $10-15$ minutes, during which the operator was asked to direct the drone using the aforementioned movements in a specific order. Each data record consists of 8 values from the sEMG signal and 9 from the ROV's inertial sensors, leading to 17 signals in total. On average, the number of data records collected from each subject was around 5500. In total, we collected and constructed a dataset consisting of 33141 records of data, labelled as a normal or abnormal movement depending on the operator and the way that each movement was executed.

\section{B. sEMG Signal Acquisition}

We used a wireless armband called Myo [10] for sEMG signal acquisition, that uses 8 EMG active electrodes and can detect sEMG data just by placing it on a person's forearm. The Myo armband transmits the data over bluetooth to any host computer, and also provides raw sEMG data from the human operator. We sampled the sEMG signals at $200 \mathrm{~Hz}$.

\section{ROV's Data Acquisition}

To collect the ROV data, we used a drone, namely DJI Spark [11]. During each flight, using the DJI Assistant Software, we collected the raw inertial sensor data for all the flights performed. Our objective was to utilize sensors which reside on the drone, eliminating the need for external data or sensors such as GPS. The onboard inertial sensors used consist of an accelerometer, a gyroscope and a magnetometer. The accelerometer measures the acceleration and enables the system to know the rate of change of velocity of the drone in the three axes. The gyroscope measures the rotational motion (in three axes) in order to perceive the full rotational movements of the drone handle titling caused by air. Finally, the magnetometer identifies the changes to the orientation of the drone and helps restore the drone to its original intended direction [2]. In this work, we did not utilize the altimeter sensor, as we focused on on-air movements; we plan to do so in future works. To ensure the safety of the operator and the drone, an experienced pilot was present at all times. We also sampled the ROV's data at $200 \mathrm{~Hz}$.

\section{Data Fusion}

After collecting both sEMG and ROV's signals for the construction of the fused dataset, we use the timestamp of both signals to match the collected signals and fused them together into a single data stream. In total 8 sEMG signals were acquired and fused with the 9 values collected from accelerometer $(\mathrm{x}, \mathrm{y}, \mathrm{z})$, gyroscope $(\mathrm{x}, \mathrm{y}, \mathrm{z})$ and magnetometer $(\mathrm{x}, \mathrm{y}, \mathrm{z})$. Furthermore, the collected data were manually annotated as normal or abnormal movements, based on whether the participant was an experienced or non-experienced operator and the way that each movement was performed according to the intentions of the pilot. To validate our experiments and prevent any problem with the classification, we used two cameras and recorded the hands of the operator, and a panoramic view of each flight that showed the drone and the operator. By analyzing the videos, we were able to identify time-frames where the operator's movements were unstable or unintentional, which we classified as abnormal (i.e. was not operating the ROV as instructed).

Next, we investigated some dimensionality reduction techniques through feature extraction and component analysis to reduce the data processed by the classifier and, enable real-time monitoring and controlling of a device, using an on-board embedded platform.

\section{FeAture Extraction AND Dimensionality REDUCTION TECHNIQUES}

\section{A. Feature Extraction}

The process of classifying an EMG signal can be divided into feature extraction and feature or dimensionality reduction. Different studies on sEMG classification propose a variety of time-domain features. We experimented with several different features, presented in Table 1. After conducting a statistical t-test and calculating the average value for each feature, we selected only the 5 most promising time-domain features that showed a significant difference between normal and abnormal movements in order to be able to classify the movements even with the reduced number of signals. We avoid the use of frequency domain features due to the high dimensional feature vectors that are produced from these methods. The time-domain features extracted from the sEMG raw data are the Variance (VAR), Integrated Absolute Value (IAV), Maximum (MAX), Simple Square Integral (SSI) and Waveform Length (WL). We also extracted features from the ROV's data, which consist of the Median, Mean, Maximum and Minimum out of all parameters.

To this end, we present different time-domain features extracted directly from the collected raw signals of both sEMG and ROV, without the need of transformation or any other analysis. Moreover, using a window of 10 sequential 


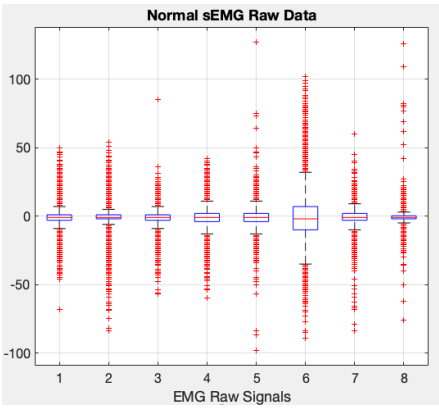

(a)

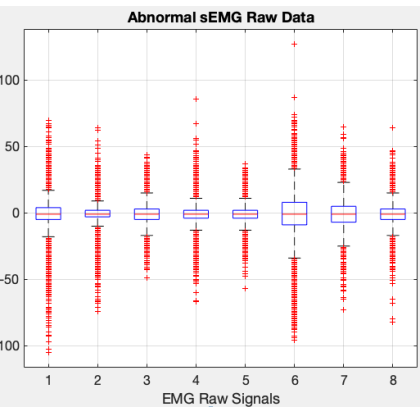

(b)

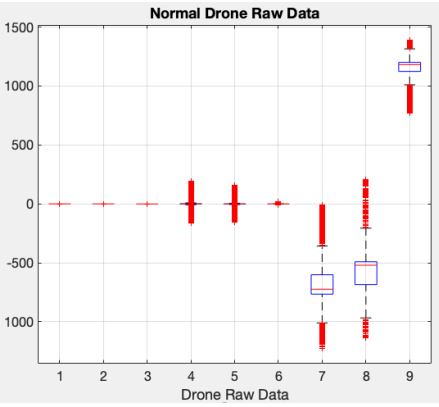

(c)

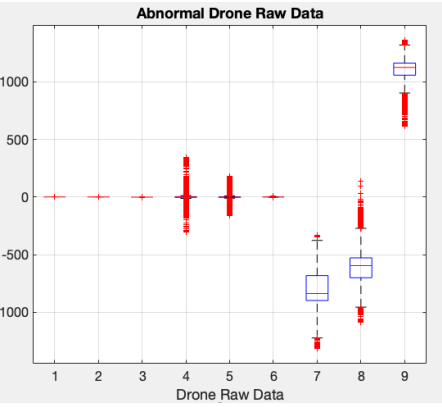

(d)

Fig. 1: Boxplots for sEMG and ROV raw signals

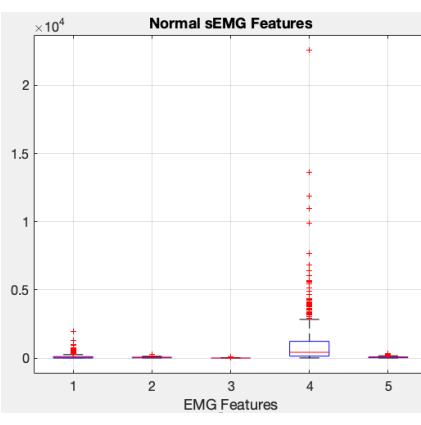

(a)

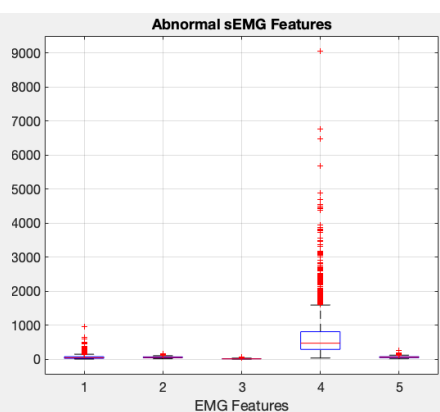

(b)

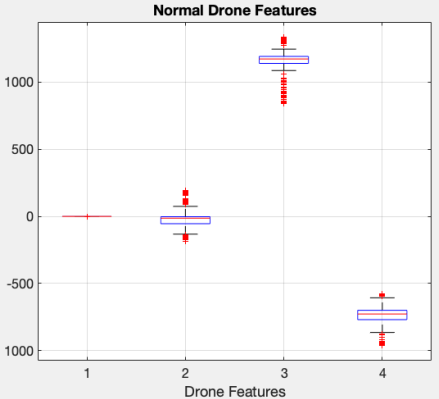

(c)

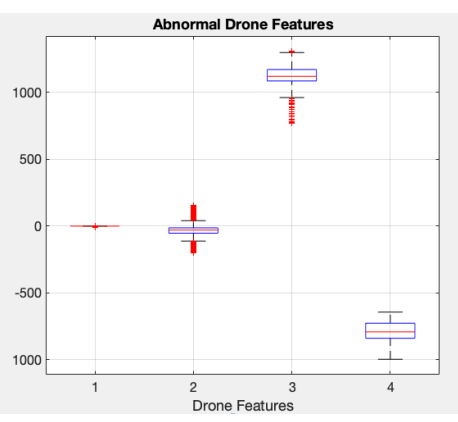

(d)

Fig. 2: Boxplots for sEMG and ROV features extracted from the raw signals

time-frames, we characterize each feature using the equations described bellow:

- Eq. (1) shows the variance, which is the average of the squares from the mean value.

$$
V A R=\frac{1}{N-1} \sum_{i=1}^{N} x_{i}{ }^{2}
$$

- Eq. (2) shows the integrated absolute value which is the absolute value of the summation of a set of values.

$$
I A V=\sum_{i=1}^{N}\left|x_{i}\right|
$$

- We also used the maximum amplitude feature which is the maximum value of a signal's amplitude in a set of values.

- Eq. (3) shows the waveform length which measures the signal complexity in a set of values.

$$
W L=\sum_{i=1}^{N-1}\left|x_{i+1}-x_{i}\right|
$$

- Eq. (4) shows the simple square integral which is the summation of the squares for each value in a set.

$$
S S I=\sum_{i=1}^{N} x_{i}{ }^{2}
$$

\section{B. Dimensionality Reduction Techniques}

We then used two well known dimensionality reduction techniques: (a) Principal Component Analysis (PCA) and (b) Independent Component Analysis (ICA). They are both statistical component analysis techniques where PCA is a statistical procedure that uses an orthogonal transform to convert a set of observations of possibly correlated variables into a set of values of linearly uncorrelated variables called principal components [12]. The principal components are linear combinations of the observed variables. On the other hand, ICA finds the independent components [13]. To this end, we use PCA and ICA to reduce the number of signals for both sEMG and ROV data and explored the impact on the performance for each classifier.

\section{Results}

\section{A. Experimental Setup}

To validate our dataset, we evaluated it across multiple commonly used classifiers, all running on resource-constrained embedded platforms (all platforms capable of being easily integrated on a commercial multi-rotor drone). We evaluate all dataset optimizations and dimentionality reductions, extracting the impact on the performance, the accuracy, the processing time and the energy consumption. We show how the raw signals, the features from sEMG and ROV's signals, and finally how applying PCA and ICA directly on raw data and features to extract 3 components for sEMG and 3 components for ROV affect accuracy, processing time and energy consumption.

Recall that we are focusing for inference on the ROV using an embedded platform while maintaining the high accuracy. To this end, 3 common classification algorithms were used that have shown from the literature that they can achieve real-time classification even on low-power devices, such as Support Vector Machines (SVM), K-Nearest Neighbors 
(KNN) and Convolutional Neural Networks (CNN). A simple $\mathrm{CNN}$ was selected, consisting of 5 layers, to enable real-time classification on embedded platform. Each classifier was trained on 2 Nvidia 960GTX GPUs on the $70 \%$ of the dataset while the other $30 \%$ was used as a test set, and evaluated on different low-power devices (a laptop i5-8250 ${ }^{1}$, a Raspberry $\mathrm{Pi}^{2}$ and a Jetson $\mathrm{TX}^{3}$ ).

\section{B. Data analysis and reduction}

The first two figures, Fig. 1a and 1b show the boxplots for each signal of the sEMG, between the normal and the abnormal sEMG raw signals. As can be seen from both figures, the distribution of the values on the abnormal signals is larger, compared to the normal sEMG signals. As such, the variation of data for the abnormal signals is different, which shows that normal and abnormal movement are distinguishable. Moving to the next two figures, Fig. 1c and 1d which show the boxplots for each signal, collected from the ROV for normal and abnormal movements, we observe that there is a similar tendency as the sEMG signals. The variation is different in 5 out of 9 signals and there is also a change on the median value, which is presented as the red line inside each box plot. In fact, this is an indication that normal and abnormal signals are easily detectable in that case. Moreover, we present the boxplots from the selected features, as shown in Fig. 2, for separated sEMG signals and ROV signals. While on most of the features there is not a significant difference between the range of values (and this might have a direct impact and drop off on the accuracy of a classifier), after calculating the average value for each feature, we observed that features such as $\operatorname{VAR}(1), \operatorname{IAV}(2)$ and $\operatorname{MAX}(3)$ have significant difference when comparing normal and abnormal data.

\section{Classification Accuracy}

We perform a detailed evaluation of different configurations between raw sEMG and ROV signals, featured sEMG and ROV's signals. To this end, we used a sampling window of 10 sequential data signals for the raw data classification and the feature extraction. Within a sampling window, there are 170 floating point values for the raw data, whereas only 9 floating point values for the features.

Moreover, we also extracted (only) 6 signals using the PCA and ICA methods. Table 2 summarizes the comprehensive comparisons between different classification algorithms. We experimented with the accuracy metrics from different cases including raw data from both the human and the ROV (our base case), raw data from the human and features extracted from ROV's data, features extracted from the human and raw data of ROV and finally, features extracted from both the human and the ROV's data. The objective of our extensive analysis was done on the basis that during the inference stage, the process of feature extraction may need to run only on the remote controller/ground station, since the ROV may be

\footnotetext{
${ }^{1}$ Quad Core 3.4GHz CPU

${ }^{2}$ Quad Core 1.2GHz Broadcom 64bit CPU

${ }^{3}$ NVIDIA Pascal, 256 NVIDIA CUDA cores
}

\begin{tabular}{c|c|c|c|c|c}
\multirow{2}{*}{ Classifier } & \multicolumn{2}{|c|}{ Data } & \multicolumn{3}{|c}{ Accuracy } \\
& EMG & ROV & PCA & ICA & - \\
\hline \hline \multirow{4}{*}{ KNN } & $\mathrm{R}$ & $\mathrm{R}$ & $\mathbf{9 5 \%}$ & $\mathbf{9 5 \%}$ & $\mathbf{9 9 \%}$ \\
\cline { 2 - 6 } & $\mathrm{F}$ & $\mathrm{R}$ & $91 \%$ & $88 \%$ & $96 \%$ \\
\cline { 2 - 6 } & $\mathrm{R}$ & $\mathrm{F}$ & $88 \%$ & $92 \%$ & $93 \%$ \\
\cline { 2 - 6 } & $\mathrm{F}$ & $\mathrm{F}$ & $85 \%$ & $81 \%$ & $85 \%$ \\
\hline \hline \multirow{3}{*}{$\mathrm{SVM}$} & $\mathrm{R}$ & $\mathrm{R}$ & $76 \%$ & $73 \%$ & $80 \%$ \\
\cline { 2 - 6 } & $\mathrm{F}$ & $\mathrm{R}$ & $74 \%$ & $73 \%$ & $77 \%$ \\
\cline { 2 - 6 } & $\mathrm{R}$ & $\mathrm{F}$ & $73 \%$ & $72 \%$ & $72 \%$ \\
\cline { 2 - 6 } & $\mathrm{F}$ & $\mathrm{F}$ & $73 \%$ & $73 \%$ & $73 \%$ \\
\hline \hline \multirow{3}{*}{$\mathrm{CNN}$} & $\mathrm{R}$ & $\mathrm{R}$ & $78 \%$ & $86 \%$ & $95 \%$ \\
\cline { 2 - 6 } & $\mathrm{F}$ & $\mathrm{R}$ & $85 \%$ & $87 \%$ & $90 \%$ \\
\cline { 2 - 6 } & $\mathrm{R}$ & $\mathrm{F}$ & $86 \%$ & $87 \%$ & $90 \%$ \\
\cline { 2 - 6 } & $\mathrm{F}$ & $\mathrm{F}$ & $84 \%$ & $89 \%$ & $85 \%$
\end{tabular}

Table 2. Accuracy results on KNN, SVM and CNN classifiers ( $R$ and $F$ indicates Raw and Featured data respectively)

too resource-constrained (or vice-versa). Thus, we explored the cases where features were used in either the drone or the operator (or both).

$R$ and $F$ in Table 2 and Fig. 3, 4 indicate the Raw and Features used in each scenario evaluated. As we can see, our optimized scenario, which includes features from the human and the ROV, gives an accuracy of $85 \%$, using a KNN (regardless if ICA is used or not), while using a CNN with PCA, gives a higher classification accuracy equal to $89 \%$. The scenario where only raw data are used (i.e. our base case) outperforms the rest of the cases, with an accuracy of $95 \%$. As we mentioned earlier, the aim is real-time and resource constrained classification, so achieving considerable accuracy (albeit reduced) with significant reduction in processing time and energy consumption, is desirable.

\section{Embedded Platforms Evaluation}

To evaluate the energy and performance gains, we implemented each of the three classifiers on the three different mobile platforms. For each platform, we used a digital energy monitoring device to measure the power consumption for CNN, SVM and KNN and multiplying it with the processing time, to measure the energy consumption. Doing so, we observe that even if the accuracy of a classifier might drop in some cases up to $10 \%$, there are significant gains in the processing time and the energy consumption of the system, which shows that our optimizations are more efficient compared to outright raw data classification. Fig. 3, shows the processing time for the original dataset and the optimized dataset, measured on all the platforms. As it can be seen, in all cases, using the optimized dataset, which consist of the features of the signal on the reduction techniques of PCA and ICA, there is a large decrease of the processing time. On the $\mathrm{RPi} 3$ there is a $20 \times$ decrease on the processing time and $5 \times$ decrease of the processing on the JetsonTX2, while using either KNN or SVM. For comparison purposes, the laptop's CPU (as it is quite powerful compared to the other platforms) experiences the least gains in performance. On the other hand, as shown in Fig. 4, when it comes to the energy 


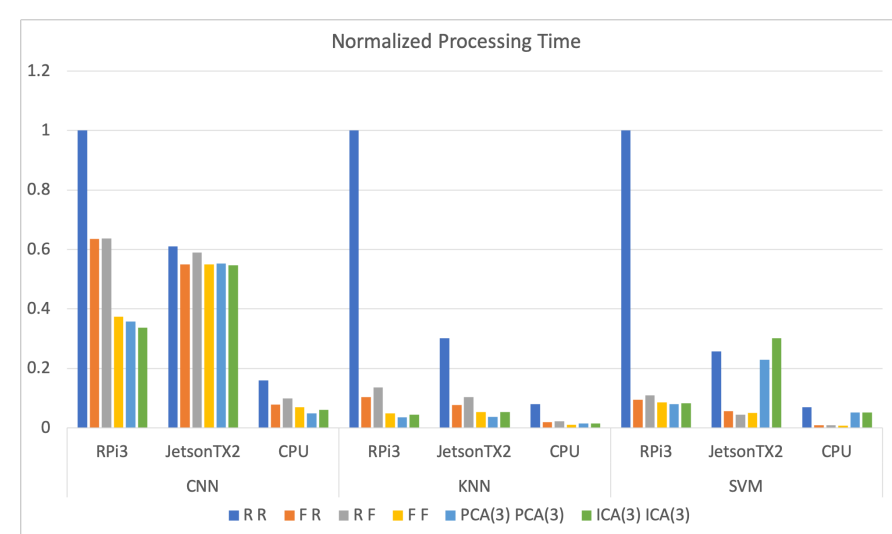

Fig. 3: Normalized Processing Time per each classifier (CNN, SVM, KNN) on each platform

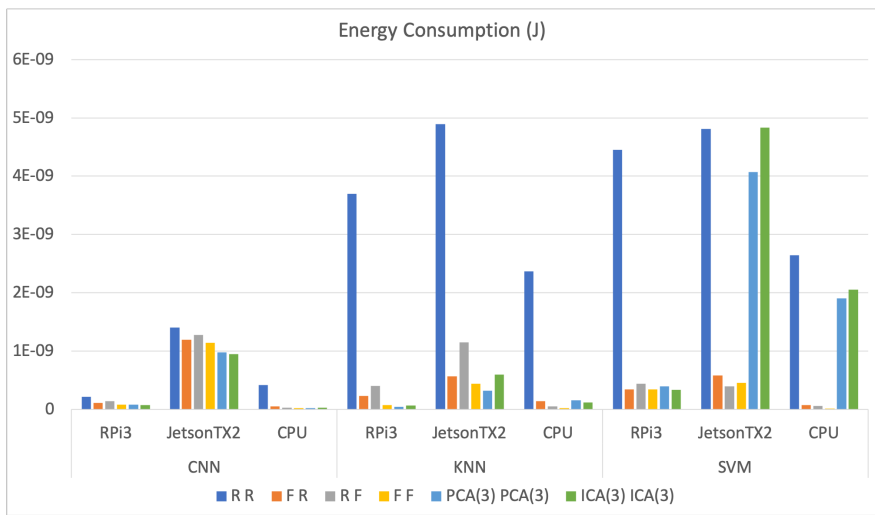

Fig. 4: Energy Consumption of CNN, SVM and KNN on each platform

consumption, the impact even on the CPU is significant. In all cases, classifying the movements with the use of features instead of raw signals will decrease at least $10 \times$ the energy consumption. It is worth mentioning that using PCA and ICA with the SVM classifier, tends to keep the processing time and the energy consumption similar to the raw signals, primarily since principal components (or independent components) are linear combinations of the original variables, so the SVM works with these combinations instead, without any significant reduction in the amount of computations.

\section{CONCLUSions AND Future Work}

Joint monitoring and controlling the human operator and an ROV during critical missions can act as a prevention mechanism for potential accidents caused by the operator's stress, inexperience and fatigue. Detecting and classifying normal and abnormal movements of the operator is an important step for such mechanism. As a first step in achieving this, we constructed a dataset consisting of sEMG signals from the human operator and inertial sensor data from an ROV. Moreover, we investigated and evaluated different features and dimensionality reduction techniques, enabling us to build a dataset geared towards real-time and energy efficient classification on a resource-constrained device. The dataset was evaluated on 3 well-known classification methods with an average drop of $10 \%$ accuracy using the optimized and reduced dataset. Moreover, we achieved an average tenfold reduction in the energy consumption leading to real-time classification when experimented on various embedded platforms. As a follow up of this work, we will focus on the design of a shared control mechanism for communication and prevention of involuntary movements, leaving the decision on the ROV, where in case of classification of abnormal movements, the ROV will choose to ignore or take corrective action. Moreover, we aim to collect more biophysical indices such as Electrocardiogram (ECG) signals and correlate the sEMG and ECG signals in an effort to increase the accuracy. In addition, we will investigate techniques to induce stress during the ROV flight. Finally, we will focus on the development of classification algorithms for the classification of such movements, an important step for the design of such system.

\section{ACKNOWLEDGEMENT}

This work has been supported by the European Union's Horizon 2020 research and innovation programme under grant agreement No 739551 (KIOS CoE) and from the Government of the Republic of Cyprus through the Directorate General for European Programmes, Coordination and Development.

\section{REFERENCES}

[1] F. Dell'Agnola, L. Cammoun, and D. Atienza, "Physiological characterization of need for assistance in rescue missions with drones," in 2018 IEEE International Conference on Consumer Electronics (ICCE), pp. 1-6, Jan 2018.

[2] G. Michael, N. Efstathiou, K. Mantis, T. Theocharides, and D. Pau, "Intelligent embedded and real-time ANN-based motor control for multi-rotor unmanned aircraft systems," in 2017 IFIP/IEEE International Conference on Very Large Scale Integration (VLSI-SoC), pp. 1-6, Oct 2017.

[3] M. Valenti, B. Bethke, J. P. How, D. P. De Farias, and J. Vian, "Embedding health management into mission tasking for UAV teams," in 2007 American Control Conference, pp. 5777-5783, IEEE, 2007.

[4] J. Healey, R. W. Picard, et al., "Detecting stress during real-world driving tasks using physiological sensors," IEEE Transactions on Intelligent Transportation Systems, vol. 6, no. 2, pp. 156-166, 2005.

[5] G. F. Wilson, "An analysis of mental workload in pilots during flight using multiple psychophysiological measures," The International Journal of Aviation Psychology, vol. 12, no. 1, pp. 3-18, 2002.

[6] M. R. Ahsan, M. I. Ibrahimy, and O. O. Khalifa, "Electromygraphy (EMG) signal based hand gesture recognition using artificial neural network (ANN)," in 2011 4th International Conference On Mechatronics (ICOM), pp. 1-6, IEEE, 2011.

[7] S. Negi, Y. Kumar, and V. M. Mishra, "Feature extraction and classification for EMG signals using linear discriminant analysis," in 2016 2nd International Conference on Advances in Computing, Communication, Automation (ICACCA) (Fall), pp. 1-6, Sep. 2016.

[8] Y. Takada, E. R. Boer, and T. Sawaragi, "Driver assist system for human-machine interaction," Cognition, Technology \& Work, vol. 19, no. 4, pp. 819-836, 2017.

[9] E. Ohn-Bar, A. Tawari, S. Martin, and M. M. Trivedi, "Predicting driver maneuvers by learning holistic features," in 2014 IEEE Intelligent Vehicles Symposium Proceedings, pp. 719-724, June 2014.

[10] M. Team, "Myo Gesture Control Armband." https://support.getmyo.com/hc/en-us/articles/ 202648103-Myo-Gesture-Control-Armband-tech-specs, 2018.

[11] DJI, "DJI Spark." https://www.dji.com/spark, 2019.

[12] H. Abdi and L. J. Williams, "Principal component analysis," Wiley Interdisciplinary Reviews: Computational Statistics, vol. 2, no. 4, pp. 433-459, 2010.

[13] G. Wang, Q. Ding, and Z. Hou, "Independent component analysis and its applications in signal processing for analytical chemistry," TrAC Trends in Analytical Chemistry, vol. 27, no. 4, pp. 368 - 376, 2008. 